\title{
Size Effect and Geometrical Effect of Polycrystals and Thin Film/Substrate System in Micro-indentation Test
}

Y. Wei, M. Zhao, X. Wang, S. Tang

LNM, Institute of Mechanics, Chinese Academy of Sciences, Beijing 100080, China

\begin{abstract}
Micro-indentation test at scales on the order of sub-micron has shown that the measured hardness increases strongly with decreasing indent depth or indent size, which is frequently referred to as the size effect. Simultaneously, at micron or sub-micron scale, the material microstructure size also has an important influence on the measured hardness. This kind of effect, such as the crystal grain size effect, thin film thickness effect, etc., is called the geometrical effect. In the present research, in order to investigate the size effect and the geometrical effect, the micro-indentation experiments are carried out respectively for single crystal copper and aluminum, for polycrystal aluminum, as well as for a thin film/substrate system, $\mathrm{Ti} / \mathrm{Si}_{3} \mathrm{~N}_{4}$. The size effect and geometrical effect are displayed experimentally. Moreover, using strain gradient plasticity theory, the size effect and the geometrical effect are simulated. Through comparing experimental results with simulation results, the length-scale parameter appearing in the strain gradient theory for different cases is predicted. Furthermore, the size effect and the geometrical effect are interpreted using the geometrically necessary dislocation concept and the discrete dislocation theory.
\end{abstract}

\section{INTRODUCTION}

Indentation test is an important and effective experimental method, and has been used extensively to estimate the plastic properties of solids undergoing the plastic deformation. Through indentation test, the loading-unloading relation between hardness and indent depth is measured. Thereby, the material parameters, such as the yielding stress, strain hardening exponent, Young's modulus, etc. are estimated. Recently, with the advancement of experimental technique and measurement precision, it is possible to carry out the indentation tests at the scale levels of one micron or sub-micron for obtaining more detailed material information. Such small-scale indentation experiments are frequently referred to as micro-indentation tests (or nano-indentation tests). In the micro-indentation test, a new result, which is different from the conventional one, size-dependent hardness result has been revealed [1-5]. For metal materials, the measured hardness may double or even triple the conventional hardness as indent size (or depth) decreases to a fifth micron. Usually, the effect is referred to as the size effect. In addition, in our recent research, we found that if material was manufactured by some microstructures and if the microstructure size was comparable to the indent depth, the size-dependent hardness results mentioned above were additionally influenced by the geometrical size. We call this the geometrical effect. In the present research, micro-indentation experiments are carried out respectively for single crystal copper and aluminum, polycrystal aluminum, as well as thin film/substrate system, $\mathrm{Ti} / \mathrm{Si}_{3} \mathrm{~N}_{4}$. Specifically, in the present research, the crystal grain size of the polycrystal specimen and the thickness of the thin film are taken into account the same order as 
that the usual size effect sensitive zone has (0.1 2 micron). The size effect and geometrical effect are displayed experimentally. It is well known that the trends of both size effect and geometrical effect are at odds with the size-independence implied by conventional elastic-plastic theory. In the present research, strain gradient plasticity theory [6] will be used to simulate the size and geometrical effect. Through comparing simulation results with experimental results, the material length parameter appearing in the strain gradient plasticity theory will be predicted. Furthermore, the size effect and the geometrical effect are interpreted using the geometrically necessary dislocation concept and the discrete dislocation theory.

\section{EXPERIMENTAL METHOD AND RESULTS}

The experimental researches are carried out in detail respectively for single crystal copper and aluminum, polycrystal aluminum, as well as thin film/substrate system $\mathrm{Ti} / \mathrm{Si}_{3} \mathrm{~N}_{4}$ on the test instrument MTS-Nanoindenter XP. In conventional indentation experiment, the indentation hardness curve is measured based on a unique loading point on specimen surface (continuous stiffness method). The hardness-indent depth curve is quite smooth. However, such a hardness curve should be dependent of the loading point status on the surface (defects or reinforced particles, etc.). In view of the reason, in the present experiments for single crystal metal and for polycrystal $\mathrm{Al}$, we use a different method by randomly selecting many loading points on specimen surface, and each loading point corresponds to only one value measured for the hardness/depth relation. Thus, a data strip about the relation is obtained. Based on the data strip, a region of the micro-scale parameter can be determined in abundance considering surface defect influences. Moreover, for comparison, in the experiment for thin film/substrate system, Ti/Si ${ }_{3} \mathrm{~N}_{4}$, we use the continuous stiffness method. The smooth hardness curves are obtained.

Figure 1(a) shows the experimental results of hardness-depth relation for single crystal copper along the (110) surface. Strip results are obtained from randomly selecting the loading points on the specimen surface. For a same depth indenting, a higher hardness value data corresponds to a strong region indented, while a lower hardness value corresponds to a weak region indented. From Figure1 (a), size effects are clearly shown, which are characterized by the hardness data trend elevating with decreasing depth. For comparison, Figure 1(b) shows another experimental results for single crystal copper along the (111) surface from [2]. From Figure 1, the hardness increases from 400nm sharply with decreasing the indent depth. Alternatively, with indent depth increasing, the hardness values tends to the stable values, conventional material hardness, i.e., triple the yielding stress for the lower-hardening metal materials.

Figure 2(a), (b) and (c) show the experimental results of the hardness-depth relations for polycrystal aluminum for different grain size cases. A series of grain sizes, $t=600 \mathrm{~nm}, 1000 \mathrm{~nm}$ and $2000 \mathrm{~nm}$, at the same order as the size effect sensitive region, are investigated. In doing each experiment, maximum indent depth is controlled to be smaller than the grain size. For comparison, Figure 2(d) gives the results for the case of single crystal Al. Features shown in Figure 2 can be described that when indent depth is small and as indent depth increases, the hardness decreases, until to some depth, the hardness tends to increase. The hardness-depth curve looks like a "U-type" shape. With further increasing crystal grain size, the U-type relation approaches a "L-type" strip, i.e., single crystal case (Figure 2(d)). The high hardness value corresponding to the small indent depth is the size effect prevailed, while the second elevation with increasing depth is the grain size effect, i.e., geometrical effect prevailed. From Figure 2, hardness-depth relation behaves like a minimum limit taken at about the indent depth reaching

W8.16.2 
one-third the crystal grain size.

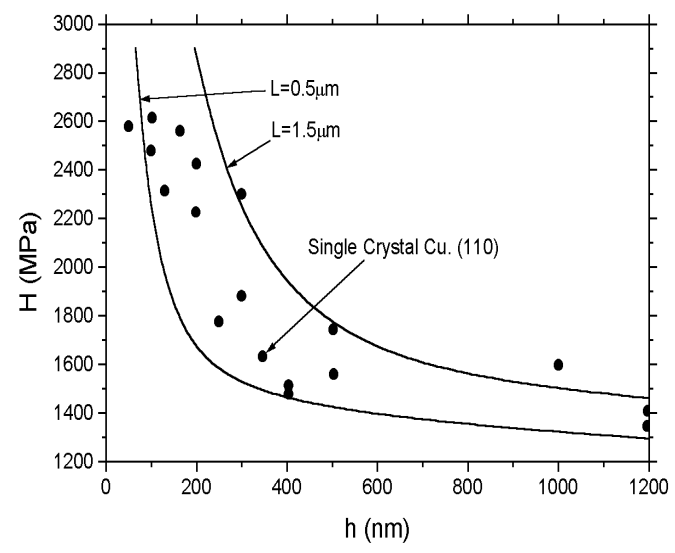

(a)

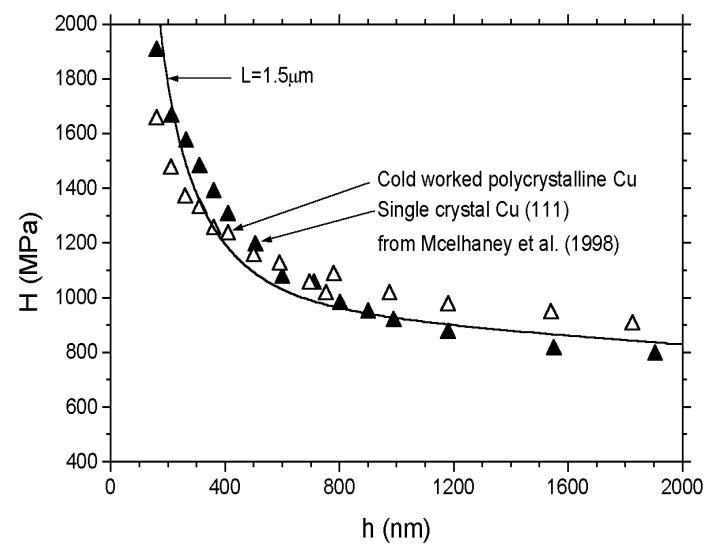

(b)

Figure 1. Hardness-depth results for single crystal copper. (a) Randomly selecting loading points. (b) Continuous stiffness method loading from [2]. Experiment and simulation results are shown.

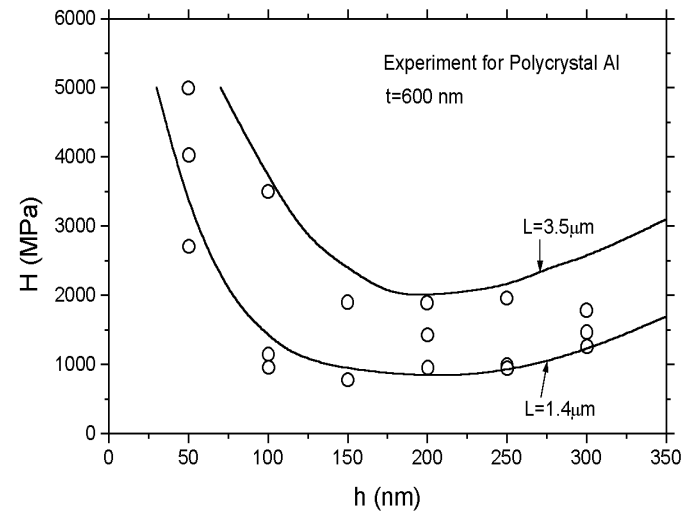

(a)

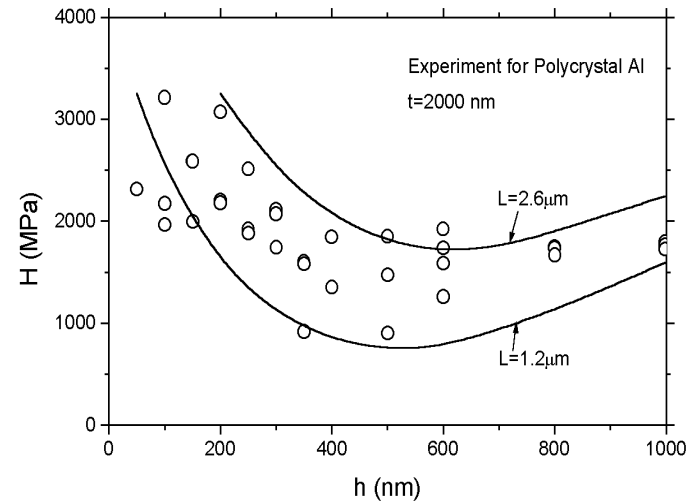

(c)

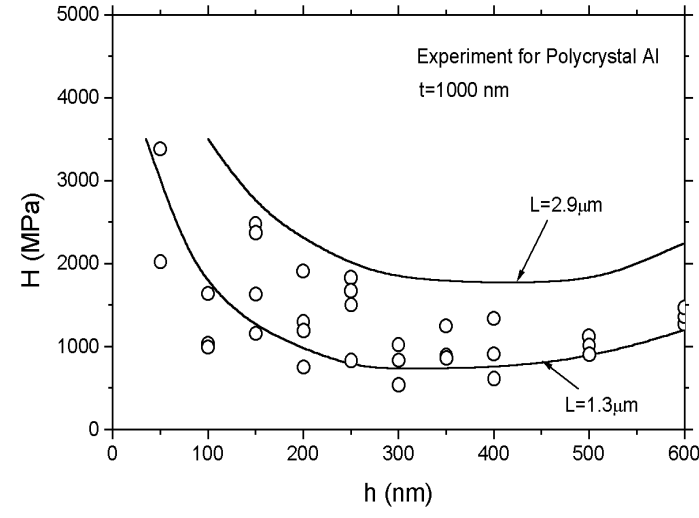

(b)

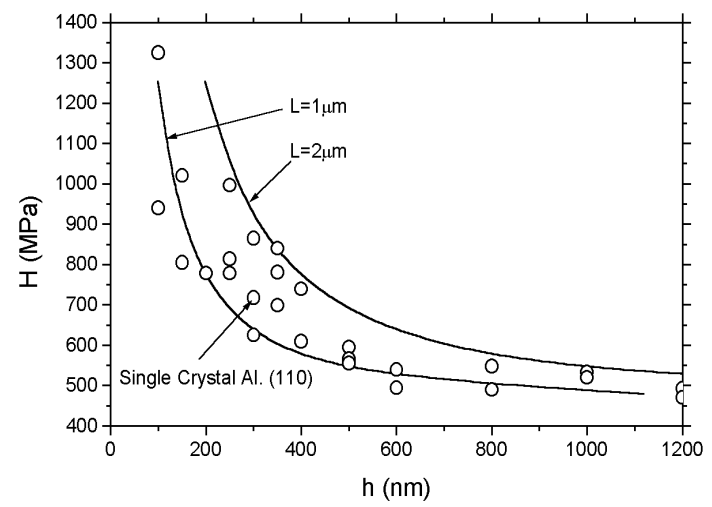

(d)

Figure 2. Hardness-depth results of polycrystal $\mathrm{Al}$ for three different grain sizes $(\mathrm{a}, \mathrm{b}$ and $\mathrm{c})$ and for single crystal $\mathrm{Al}(\mathrm{d})$. Experimental and simulation results are shown. 


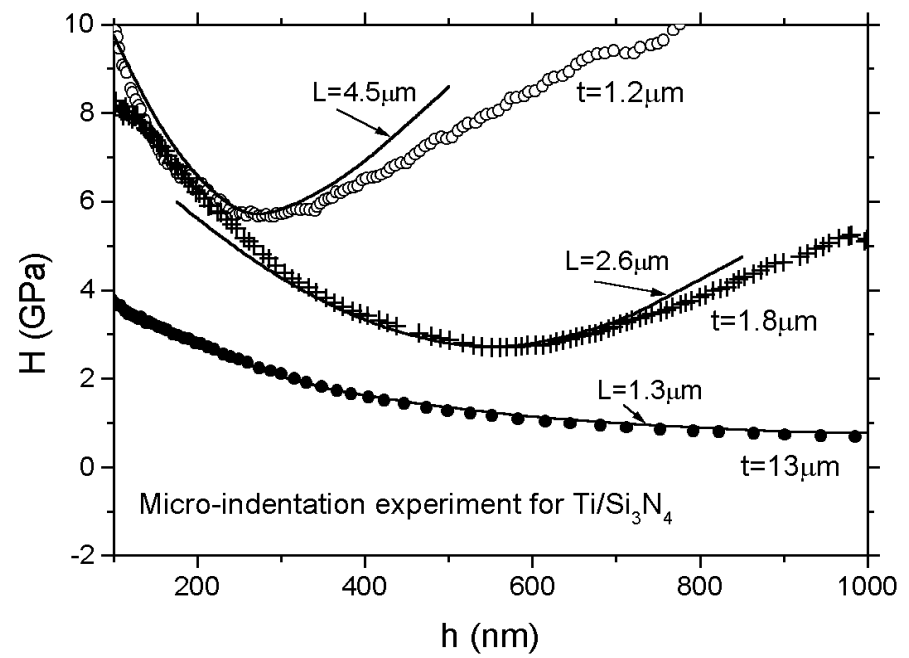

Figure 3. Hardness-depth results for thin film/substrate system for three different thickness cases. Experimental results (continuous stiffness method loading) and simulation results are shown.

Figure 3 shows the micro-indentation experimental results for a metal thin film/ceramic substrate system, $\mathrm{Ti} / \mathrm{Si}_{3} \mathrm{~N}_{4}$. A series of thin film thicknesses, $\mathrm{t}=1.2,1.8$ and 13 micrometer are taken into account in the experiments. Here, the continuous stiffness method is adopted in the indentation experiments, therefore the experimental results are quite smooth curves. Similarly, the maximum indent depth is controlled to be smaller than thickness of film for each case. From Figure 3, the hardness-depth curve looks also like the U-type shape, similar to the case of polycrystal metal just discussed above. From Figure 3, when indent depth is small, due to strong size effect the hardness value is big. With increasing the indent depth, size effect drops and hardness decreases. After the indent depth increases over critical point, about one-third of thickness of film, geometrical effect is prevailed and hardness tends to increase. When thin film thickness is very large, $\mathrm{t}=13$ micrometer, within the size effect sensitive region the hardness decreases with increasing thin film thickness monotonically.

From Figure 2 and 3, the geometrical effect (grain size effect and film thickness effect) makes the hardness curve elevate within the size effect sensitive region.

\section{NUMERICAL SIMULATION OF SIZE AND GEOMETRICAL EFFECTS}

The micro-indentation tests of polycrystal metal and metal thin film/ceramic substrate system can be simplified as the analytical models as shown in Figure 4 (a) and (b). For polycrystal material, when the instrument indenter acts at a crystal grain on specimen surface, with indent depth increase, a plastic zone develops within the grain and is limited by grain boundary, considering that indent depth is smaller than grain size. Similar simplification consideration is for the thin film/substrate system.

On the simulation of the size effect and the geometrical effect, one can't use the conventional elastic-plastic theory, because no length parameter is included to distinguish a macro-scale problem with a micro-scale problem. To predict the effects, strain gradient plasticity theories are 


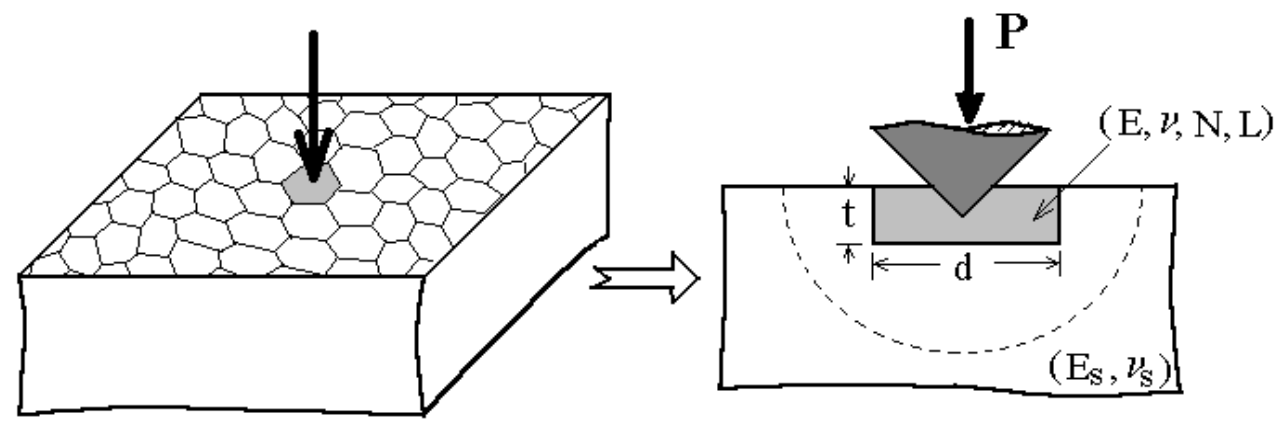

(a)

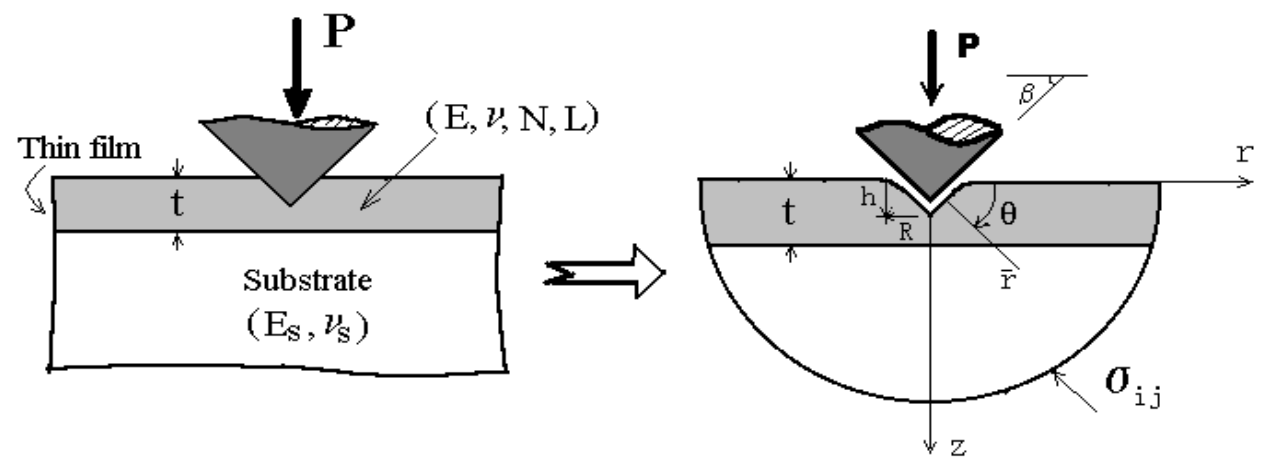

(b)

Figure 4. Simplified models. (a) Polycrystal metal undergoing the indenting, and (b) metal thin film/ceramic substrate system undergoing the indenting.

prevailed. Several version strain gradient theories have been presented, e.g. [6-8]. In the present research, Fleck and Hutchinson strain gradient theory will be adopted [6,9]. The deformation theory of Fleck and Hutchinson strain gradient plasticity can be dictated as follows for the constitutive equations (relations of stress and higher stress versus strain and higher strain) [5],

$$
\begin{gathered}
\sigma_{i j}=\frac{E}{1+v+\frac{3}{2} E / h^{p}} \varepsilon_{i j}+\frac{1}{3}\left(\frac{E}{1-2 v}-\frac{E}{1+v+\frac{3}{2} E / h^{p}}\right) \varepsilon_{k k} \delta_{i j} \\
\tau_{i j k}=2 E\left\{\sum_{I=1}^{3} \frac{L_{I}^{2}}{L_{I}^{2} / L_{I}^{e 2}+2 E / h^{p}} T_{i j k l m n}^{(I)}+L_{4}^{e 2} T_{i j k l m n}^{(4)}\right\} \eta_{l m n}
\end{gathered}
$$

where $L_{I}^{e}$ and $L_{I}(I=1,4)$ are the micro-scale parameters of elastic and plastic case, respectively. From the discussion in [6], there exists a general relation among the micro-scales (SG theory):

$$
L_{1}=L, \quad L_{2}=\frac{1}{2} L, \quad L_{3}=\sqrt{\frac{5}{24}} L
$$

In addition, take $L_{4}=L / 2$. Similarly, (2) is also valid for strain gradient elasticity lengths, replacing $L$ by $L^{e}$ in form. Moreover, previous research has shown that the solution is insensitive to the value of $L^{e} / L$ within the region $0<L^{e} / L<1$ [9]. Thus, take $L^{e} / L=0.5$ in the present research. 
In equation (1), $h^{p}$ is the effective plastic modulus, its expression depends on the material tensile stress-strain curve. In the present research, conventional power-law hardening material is considered. Finite element method is used to simulate the size effect and geometrical effect [5].

In our simulation, take several material parameters as $E / \sigma_{Y}=300, v=v_{s}=0.3, N=0.1$, and $E_{s} / E=1$ for single- and poly-crystal metal, as well as $E_{s} / E=6$ for thin film/ceramic system. Analysis models are shown in Figure 4(a) and (b). The simulation results have been shown in Figures 1-3, together with experimental results, see solid lines. From the figures, clearly, simulation results using strain gradient plasticity theory capture the experimental trends. Comparing the simulation results with corresponding experimental results, one can obtain the length parameter values. For single crystal metals, from Figure 1 and Figure 2(d), the material length parameter value is around one micron. For polycrystal and thin film/substrate system, as shown in Figure 2 and Figure 3, considering microstructure size effects (geometrical effect), the length parameter value increases, depending on the microstructure size.

Size effect has been connected with the density of geometrically necessary dislocation (DGND) at microscale [4, 10]. However, the geometrical effect case seems more complicated than the size effect case. Besides the DGND factor, in the geometrical case the blocking of grain boundary or interface to dislocation moving must be a main factor. Besides adopting the strain gradient plasticity theory, one can use the discrete dislocation method to simulate the effect [11].

\section{CONCLUSIONS}

Size effect and geometrical effect have been studied experimentally and numerically in the micro-indentation test. Both effects have been displayed clearly for the single crystal metals, polycrystal metal, as well as the metal thin film/ceramic substrate system in the experimental results. The numerical simulation based on the strain gradient plasticity theory has captured the experimental phenomena. Through comparing the experimental results with simulation results, material length parameter appearing in the strain gradient plasticity theory has been predicted.

\section{ACKNOWLEDGMENT}

This work was supported by the National Natural Science Foundation of China through Grants 19891180 and 19925211, and jointly by "Bai Ren Plan" of Chinese Academy of Sciences.

\section{REFERENCES}

1. Q. Ma and D.R. Clarke, J. Mater. Res. 10, 853 (1995).

2. K.W. McElhaney, J.J. Vlassak and W.D. Nix, J. Mater. Res. 13, 1300 (1998).

3. M.Begley, J.W. Hutchinson, J. Mech. Phys. Solids 46, 1029 (1998).

4. W.D. Nix and H. Gao, J. Mech. Phys. Solids 46, 411 (1998).

5. Y. Wei, X. Wang, X. Wu and Y. Bai, Science in China (Series A) 44, 74 (2001).

6. N.A. Fleck and J.W. Hutchinson, Advances in Applied Mechanics 33, 295 (1997).

7. H. Gao, Y. Huang, W.D. Nix and J.W. Hutchinson, J. Mech. Phys. Solids 47, 1239 (1999).

8. E.C. Aifantis, Int. J. Eng. Sci. 30, 1279 (1992).

9. Y. Wei, J.W. Hutchinson, J. Mech. Phys. Solids 45, 1253 (1997).

10. N.A. Fleck, G.M. Muller, M.F. Ashby and J.W. Hutchinson, Acta Met. Mat. 42, 475(1994).

11. J.Y. Shu, N.A. Fleck, E.V. Giessen and A. Needleman, J. Mech. Phys. Solid 49, 1361(2001).

W8.16.6 